\title{
Neoumbilicoplastia como opção de reconstrução umbilical nas dermolipectomias abdominais em âncora pós-gastroplastia
}

\author{
Neo-umbilicoplasty as an option in umbilical reconstruction in abdominal anchor \\ dermolipectomy post gastroplasty
}

ERnando Luiz Ferraz

Cavalcanti $^{1}$

Trabalho realizado no Hospital Universitário Oswaldo Cruz -

Universidade de Pernambuco

- Serviço de Cirurgia Plástica

Reconstrutora e do Ex-obeso, Recife, PE, Brasil.

Trabalho apresentado e aprovado no Exame para Ascensão a Membro Titular da SBCP, realizado no $46^{\circ}$ Congresso Brasileiro de Cirurgia Plástica.

Artigo submetido pelo SGP (Sistema de Gestão de Publicações) da RBCP.

Artigo recebido: $27 / 6 / 2010$ Artigo aceito: 26/7/2010

\begin{abstract}
RESUMO
Introdução: A abdominoplastia em âncora após grande perda ponderal secundária à cirurgia bariátrica está cada vez mais sendo realizada em nossa especialidade. Ao mesmo tempo, o surgimento de novas técnicas de reconstrução umbilical, bem como a evolução das já existentes, vem sempre acrescentar ao arsenal cirúrgico da especialidade, no intuito de promover melhores índices de satisfação e menores complicações. Método: $\mathrm{O}$ trabalho do tipo série de casos tem como objetivo principal descrever os resultados obtidos com as umbilicoplastias realizadas nas abdominoplastias em âncora em 31 pacientes, no perído de janeiro de 2008 a julho de 2009, apresentando detalhamento técnico de neoumbilicoplastia. Como objetivos secundários são descritas as características gerais e transoperatórias da população estudada. As variáveis idade, sexo, IMC, incidência de hérnias, peso da peça, tempo cirúrgico e complicações foram analisadas. Resultados: Os resultados demonstram que as características gerais, dados transoperatórios e complicações são compatíveis com outros trabalhos publicados na literatura. Conclusão: A técnica de neoumbilicoplastia descrita se mostrou um método de reconstrução umbilical de fácil realização e com melhores resultados estéticos que a tática de umbilicoplastia por transposição, com menor incidência de complicações, importante satisfação do autor e dos pacientes. Entretanto, um estudo analítico em longo prazo seria necessário para que esses resultados possam ser extrapolados para o universo dos pacientes ex-obesos.
\end{abstract}

Descritores: Abdome/cirurgia. Umbigo/cirurgia. Gastroplastia. Perda de peso.

\section{SUMMARY}

Introduction: Anchor-line abdominoplasty after massive weight loss secondary to bariatric surgeries are increasingly being performed in our specialty. At the same time, the emergence of new techniques for umbilical reconstruction, as well as the evolution of existing ones has always add to the armamentarium of surgical specialty, in order to promote best levels of satisfaction and fewer complications. Methods: This work of series of cases aims to describe the main results of the umbilicoplasty held at anchor-line abdominoplasty in 31 patients from January 2008 until July 2009, presenting technical details of neo-umbilicoplasty. As a secondary objective, describes the general characteristics and trans-operative population. Variables analised: age, sex, BMI, incidence of hernias, part weight, duration of surgery and complications. Results: The results show that the general characteristics, trans-operative data and complications are consistent with other published studies in the literature. Conclusions: The technique of neo-umbilicoplasty proved to be an easy method of umbilical reconstruction with better cosmetic results than the tactic of umbilicoplasty for transposition, with fewer complications, a great satisfaction of the author and the patients. However, an analytical study on long-term would be necessary for these results can be extrapolated to the universe of ex-obese patients.

Descriptors: Abdomen/surgery. Umbilicus/surgery. Gastroplasty. Weight loss.

1. Membro Titular da Sociedade Brasileira de Cirurgia Plástica; Cirurgião Plástico do Serviço de Cirurgia Plástica do IMIP e do Hospital Universitário Oswaldo Cruz. 


\section{INTRODUÇÃO}

As cirurgias plásticas após grandes perdas ponderais secundárias às cirurgias bariátricas estão cada vez mais sendo realizadas em nossa especialidade, e, em meio às mais variadas técnicas cirúrgicas sobre o tema, destacamse as abdominoplastias verticais modificadas ${ }^{1}$, como a abdominoplastia em âncora, indicada em casos selecionados onde há excesso cutâneo horizontal e vertical no abdome ${ }^{2}$.

As umbilicoplastias, mesmo em cirurgias convencionais, por muitas vezes são o ponto fraco dos resultados das abdominoplastias. Dessa forma, alguns trabalhos ao longo de décadas foram publicados na tentativa de se obter um melhor resultado estético ${ }^{3-5}$, tanto com técnicas de isolamento e transposição ${ }^{6}$, como com técnicas de neoumbilicoplastia ou reconstrução umbilical ${ }^{7-9}$, associadas ou não à presença da cicatriz mediana ${ }^{10,11}$.

Em se tratando de pacientes ex-obesos, quase a totalidade das cicatrizes umbilicais isoladas durante as abdominoplastias tem pedículos muito longos, de difícil acomodação e fixação à aponeurose, gerando assim vascularização limítrofe e dificuldades na definição de sua forma. Além disso, em muitas situações, a higiene está prejudicada. Sofrimento cutâneo, deiscências, cicatrização prolongada, despigmentações, estenoses e hipertrofias cicatriciais são algumas complicações passíveis de ocorrer ${ }^{12}$, gerando, obviamente, pós-operatórios mais trabalhosos.

É de se esperar que a evolução de técnicas existentes, bem como a criação de novas ideias ou detalhes técnicos relacionados às abdominoplastias e à reconstrução umbilical nesses pacientes venham a acrescentar, na busca de melhores resultados, ao arsenal cirúrgico já existente, promovendo a satisfação de pacientes e seus cirurgiões.

O trabalho em questão é de caráter descritivo, tipo série de casos, e tem como objetivo principal descrever os resultados obtidos das umbilicoplastias realizadas em 31 pacientes submetidos a dermolipectomia abdominal em âncora pós-gastroplastia, realizados pelo autor, no período de janeiro de 2008 a julho de 2009, apresentando detalhamento técnico de neoumbilicoplastia. Como objetivo secundário, serão descritas as características gerais e transoperatórias da população estudada.

\section{MÉTODO}

Cento e vinte e seis pacientes que realizaram a cirurgia bariátrica procuraram espontaneamente o Serviço de Cirurgia Plástica para avaliação das diversas cirurgias plásticas possíveis em cada caso. Destes, 55 desejaram realizar a abdominoplastia.

Foram adotados os seguintes critérios de inclusão: índice de massa corpórea (IMC) abaixo de $35 \mathrm{~kg} / \mathrm{m}^{2}$, idade entre 18 e 60 anos, tabagismo inativo há pelo menos 30 dias, realização de gastroplastia com incisão mediana supraumbilical há pelo menos 1 ano, com estabilização de peso há pelo menos 6 meses, submetidos a dermolipectomia abdominal exclusivamente pelo autor.

Foram excluídos os pacientes com comorbidades descompensadas, como diabetes, hipertensão, coagulopatias e cardiopatias, além de nível de hemoglobina menor que $12 \mathrm{mg} / \mathrm{dl}$.

Trinta e um pacientes preencheram os critérios acima e foram submetidos ao procedimento de dermolipectomia abdominal em âncora. Em 17 (54,5\%) casos, foi realizada neoumbilicoplastia por tática a ser descrita, sendo realizada a umbilicoplastia de transposição convencional em 14 $(45,5 \%)$ pacientes.

As variáveis descritas foram: idade, sexo, IMC, incidência de hérnias, peso da peça ressecada, tempo cirúrgico e complicações encontradas.

\section{Técnica operatória}

Primeiramente, o paciente foi marcado em pé para delimitar o descolamento vertical mediano, seguido da marcação arqueada da região púbica com o paciente deitado. O ponto de confluência das duas marcações, na grande maioria das vezes, se situa abaixo do nível do umbigo (Figura 1).

Após anestesia geral ou por bloqueio, com o paciente em decúbito dorsal sem elevação do dorso, a pele foi incisada em sua linha mediana do apêndice xifóide até a marcação inferior do púbis, com transecção e desinserção do umbigo nos casos de neoumbilicoplastia, e isolamento do coto
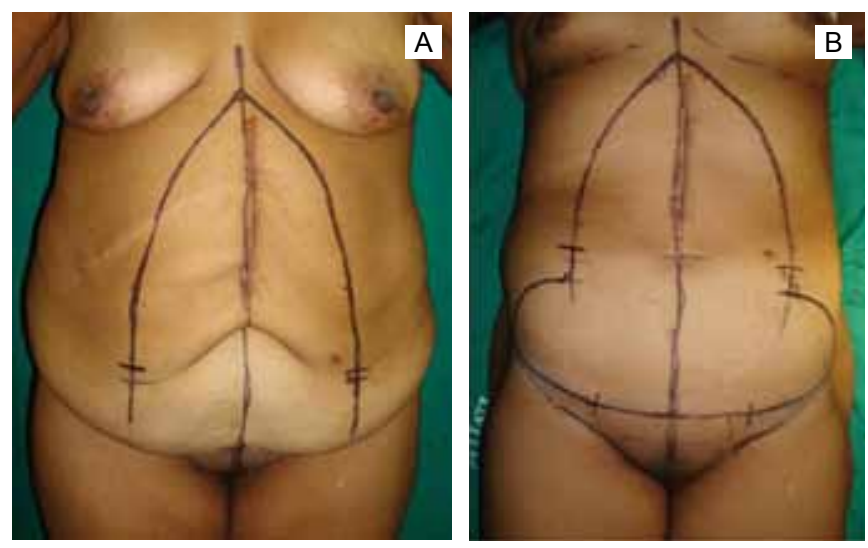

Figura 1 - Marcação dos retalhos em pé e deitado. 

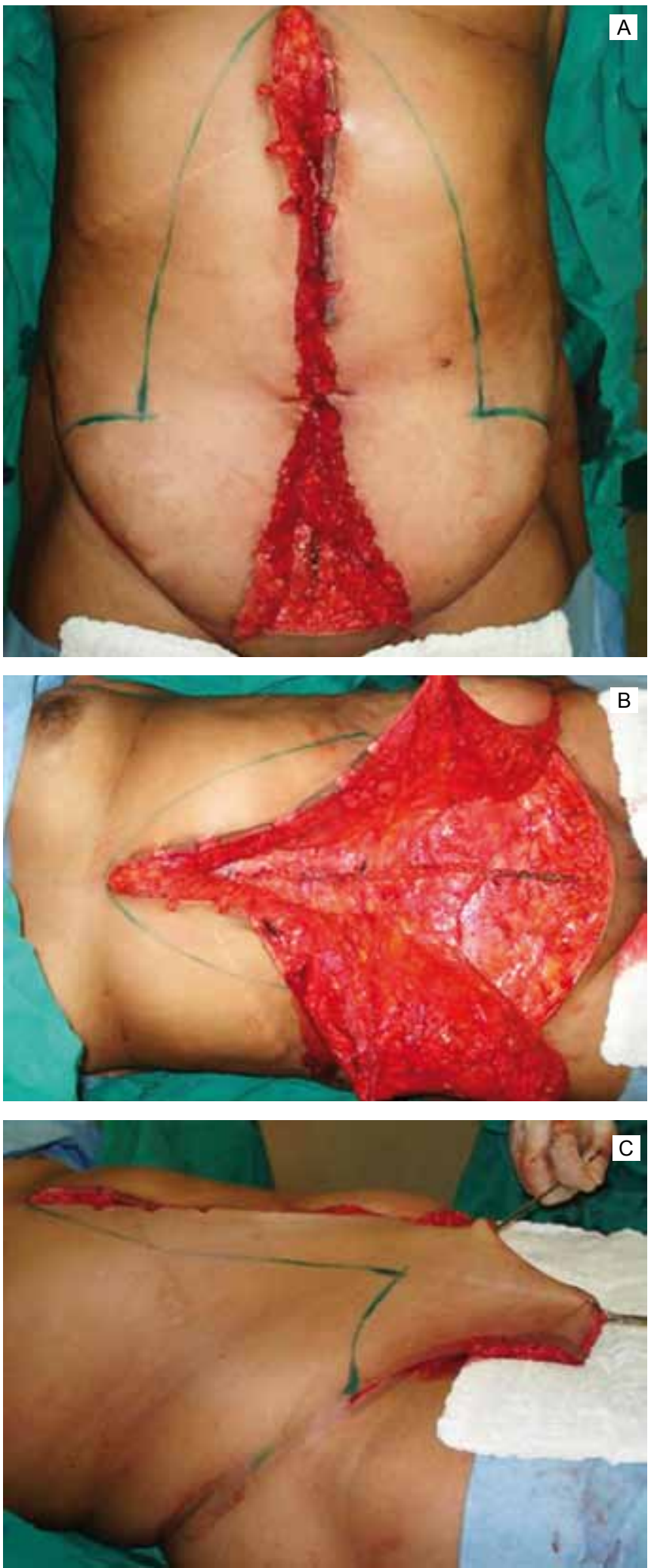

Figura 2 - A: Retalhos dermogordurosos descolados. B: Plicatura mediana realizada. $\boldsymbol{C}$ : Tração médio-inferior dos retalhos com marcação definitiva do ponto de intersecção das incisões. umbilical nos casos de transposição. O retalho dermogorduroso foi descolado lateralmente em plano supra-aponeurótico até a marcação, isolando os eventuais sacos herniários umbilicais e/ou incisionais. Em seguida, a pele foi incisada em sua marcação arqueada púbica e descolado o retalho superiormente até próximo à marcação da intersecção dos fusos vertical e horizontal (Figura 2A).

Foi realizada plicatura da aponeurose com fio de polidioxanona (PDS $\left.{ }^{\circledR}\right)$ II 1 duplo, em fuso xifo-pubiano, contínuo e trançado, reparando neste tempo as eventuais hérnias. A largura do fuso coincide com o limite do descolamento lateral dos retalhos (Figura 2B). Em 1 caso, houve necessidade de colocação de tela de polipropileno para complementar o fechamento da hérnia.

Os retalhos abdominais foram, então, tracionados medial e inferiormente em direção ao meio da incisão inferior, marcando-se definitivamente o ponto de intersecção das incisões vertical e horizontal (Figura 2C).

Nos casos em que a neoumbilicoplastia foi realizada, a cerca de $10 \mathrm{~cm}$ acima deste ponto, coincidindo com o nível das cristas ilíacas ou com o posicionamento anterior do coto umbilical amputado, na linha de incisão vertical, marcou-se 2 pequenos retalhos hexagonais de base lateral (Figura 3A). Toda a pele demarcada foi novamente incisada e retiraramse finalmente os tecidos excedentes (Figura 3B).

Esses retalhos eram exclusivamente dérmicos, já que eram desengordurados em sua base (Figura 4A). O subcutâneo abaixo do retalho não foi ressecado, e sim rebatido lateralmente, para que não faltasse preenchimento lateral ao neoumbigo, dando melhor profundidade ao mesmo (Figura 4B).

Cinco pontos com mononylon 3-0 foram necessários para a fixação dos retalhos dérmicos à aponeurose, sendo $3 \mathrm{em}$ linha média e 2 cerca de $0,5 \mathrm{~cm}$ lateralmente ao ponto central (Figuras 5 e 6A).

Nos casos em que houve preservação do coto umbilical, o pedículo foi encurtado por fixação do mesmo à aponeurose com fio inabsorvível, e a umbilicoplastia foi losangular.

A sutura do tecido gorduroso na linha média foi realizada com fio de poliglactina (vicril $\left.{ }^{\circledR}\right) 0$ ou 1 , fixando-se a fáscia de Scarpa à aponeurose, para reduzir o espaço morto subcutâneo supraumbilical. A ferida operatória foi fechada em plano dérmico profundo e superficial com mononylon 3-0 ou 4-0 e poliglecaprone (monocril ${ }^{\circledR}$ ) 4-0 (Figura 6B). Um dreno a vácuo foi posicionado no subcutâneo, ao longo da região infraumbilical.

Todos os pacientes receberam alta hospitalar no dia seguinte, sendo prescrito antibiótico por 24 horas e antiinflamatório por 5 dias. Não foi utilizada heparina como adjuvante profilático da trombose venosa nos casos descritos. 

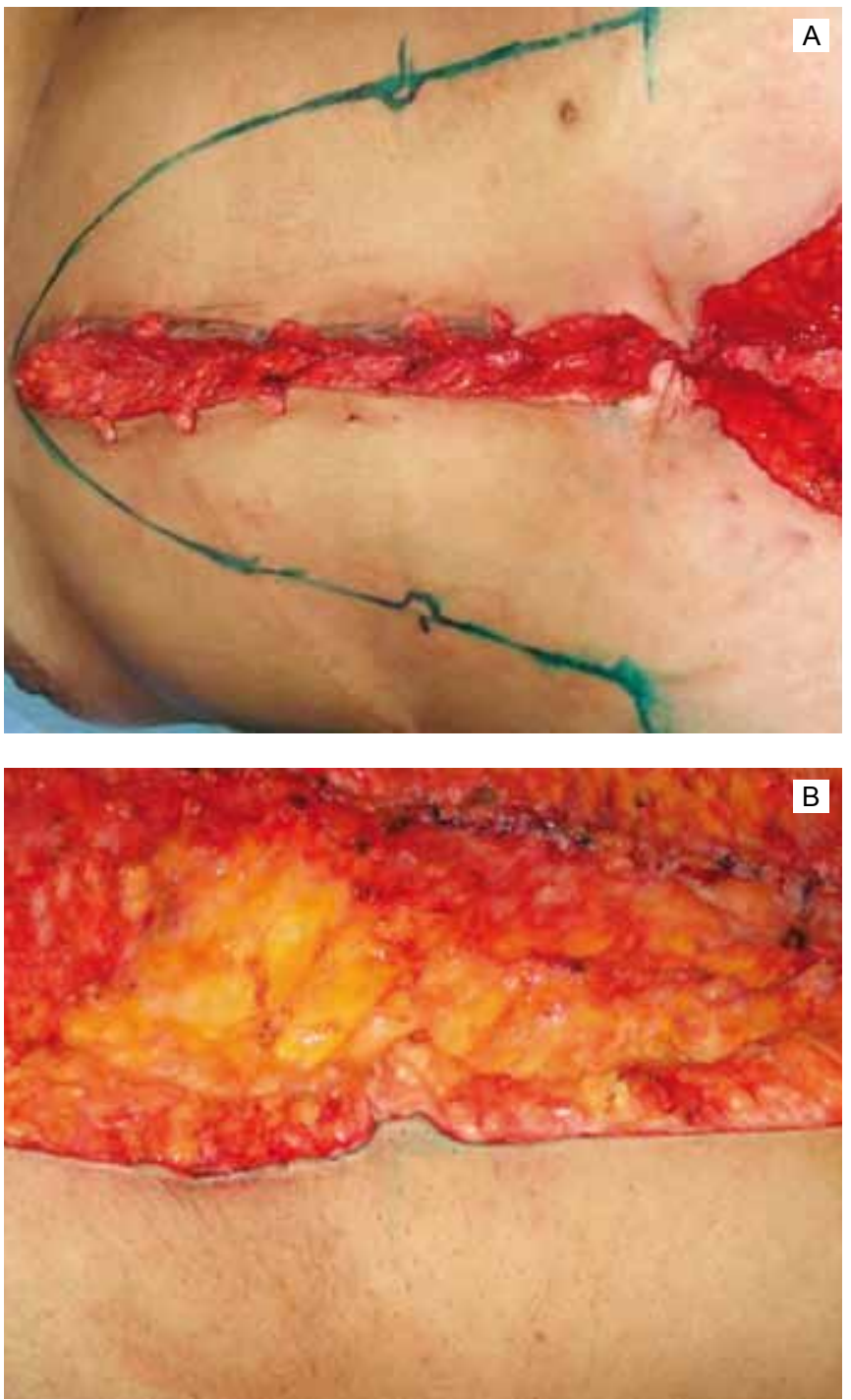

Figura 3 - A: Marcação dos retalhos do neoumbigo. B: Detalhe, após ressecção dos retalhos abdominais excedentes.

A retirada do dreno ocorreu no $4^{\circ}$ ou $5^{\circ}$ dia, ou quando o débito encontrava-se com volume inferior a $50 \mathrm{ml} \mathrm{em}$ 24 horas.

O seguimento pós-operatório foi semanal no primeiro mês e mensal nos 2 meses seguintes, além de revisão a cada 6 meses subsequentes, com registro detalhado das evoluções pós-operatórias.

A satisfação do paciente foi avaliada por meio de livres relatos quanto à beleza de seus umbigos operados, e a avaliação do autor foi subjetiva, baseada em vivência teórica e prática com umbilicoplastias em sua rotina pessoal e profissional.

Esse estudo foi aprovado em Comissão de Ética e Pesquisa, sob o número de protocolo 94.
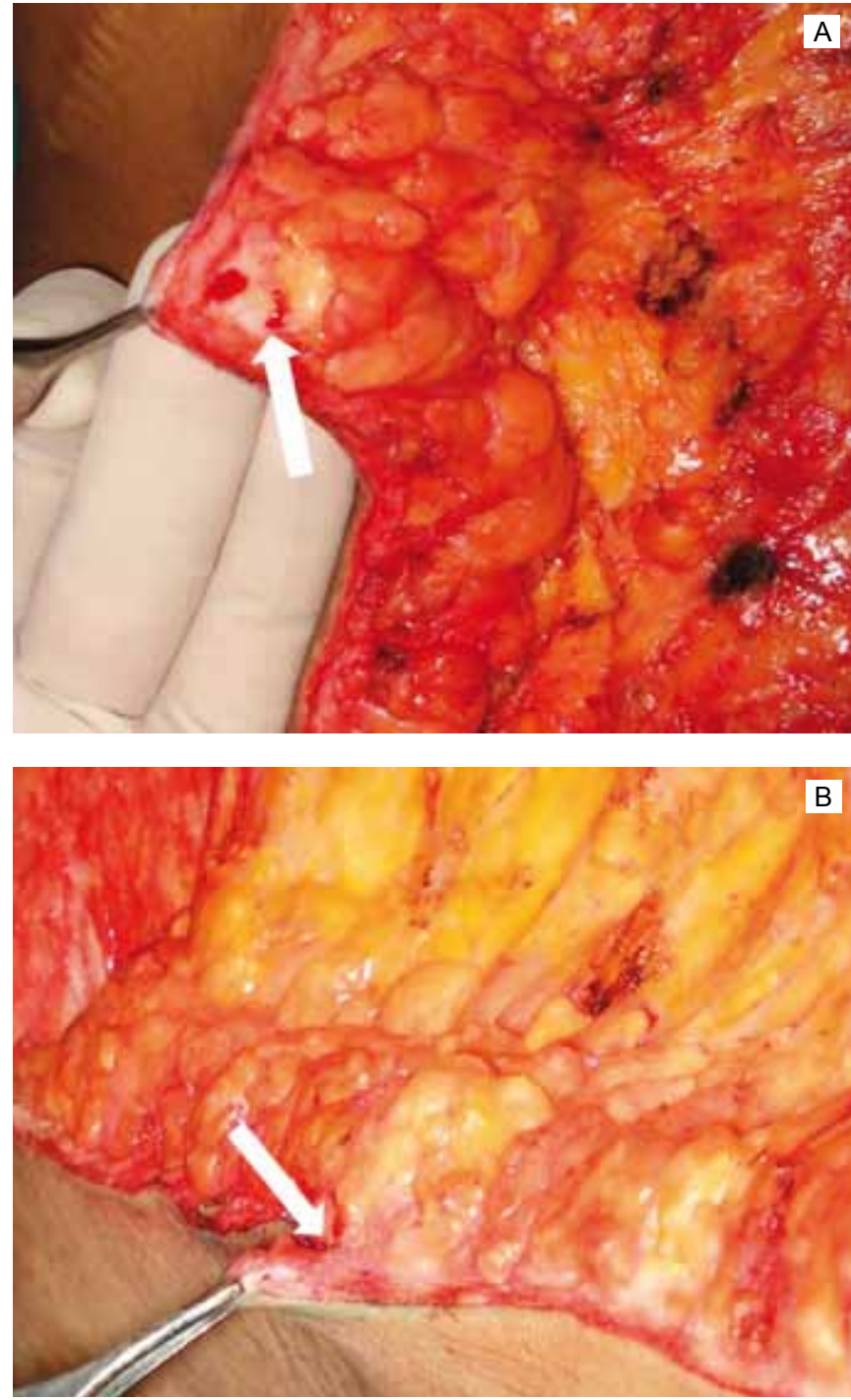

Figura 4 - Retalho do neoumbigo em destaque sob visão frontal (A) e em perfil (B). Observa-se o plano de descolamento de sua base (seta), deixando o retalho exclusivamente dérmico.

\section{RESULTADOS}

Foram operados 31 pacientes, sendo 26 (83\%) mulheres e $5(17 \%)$ homens. As características gerais da amostra estão descritas na Tabela 1, sendo separados os casos de neoumbilicoplastia das umbilicoplastias de transposição.

A Tabela 2 demonstra as variáveis transoperatórias.

O diâmetro dos sacos herniários encontrados variou de $1 \mathrm{a} 15 \mathrm{~cm}$, com média de $4,9 \mathrm{~cm}$, estando, em 2 casos, o epíplon domiciliado. $\mathrm{O}$ reparo foi realizado pela plicatura em fuso, exceto em 1 caso, no qual foi utilizada uma tela de polipropileno, devido à grande extensão lateral da falha aponeurótica. 


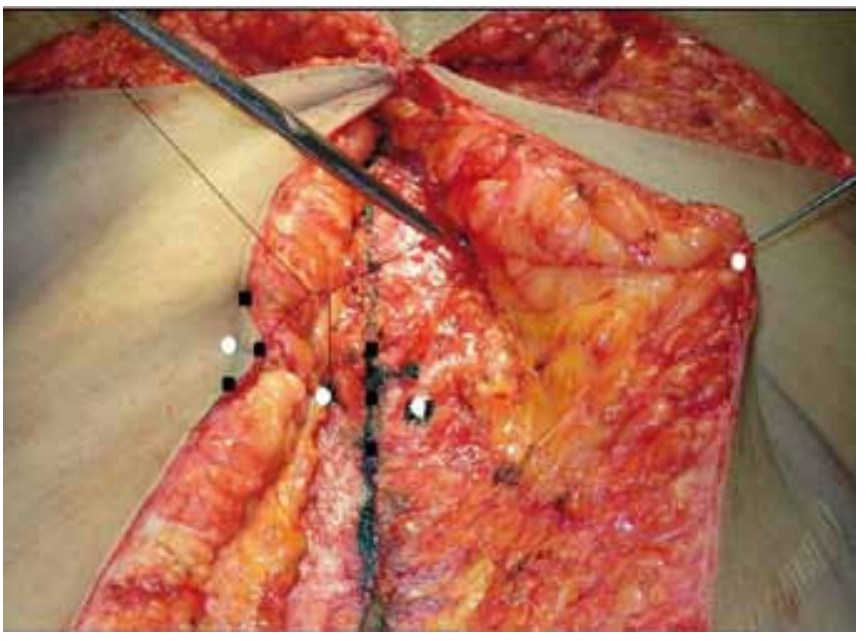

Figura 5 - Suturas de fixação do retalho do neoumbigo à aponeurose. Os circulos brancos indicam os pontos nas bases dos retalhos; os quadrados pretos os pontos da linha média.

As complicações encontradas e suas incidências são citadas na Tabela 3 .

Os seromas diagnosticados clinicamente foram puncionados sob técnica asséptica. Todas as deiscências dos casos desse estudo foram tratadas por expectação e curativos com pomadas antibióticas. As celulites encontradas, tratadas com antibiótico oral, ocorreram em 2 dos 5 homens do estudo, assim como 3 deles apresentaram seroma. $\mathrm{O}$ único hematoma importante apresentado evoluiu com celulite de $\mathrm{FO}$ e deiscência total da umbilicoplastia em losango. $O$ tratamento foi realizado com internamento, antibioticoterapia venosa e drenagem espontânea e por compressão dos coágulos através da FO. As cicatrizes com alargamento maior que $0,5 \mathrm{~cm}$ se localizavam principalmente na linha média supra-umbilical.

O tempo de evolução ou seguimento pós-operatório médio foi de 9,5 meses.

As Figuras 7 a 10 demonstram alguns dos resultados obtidos pelas técnicas citadas.

A técnica em âncora utilizada apresentou uma limitação nas ressecções dos excedentes mais laterais, porém o abdome anterior apresentou resultados bastante satisfatórios. Em 2 casos, os pacientes apresentaram maiores aventais, onde há maior presença de pregas cutâneas laterais e de abdome superior, havendo necessidade de ressecar o retalho de forma invertida concomitantemente, gerando cicatrizes também a nível de sulco inframamário.

As queixas mais relacionadas à umbilicoplastia em losango foram: cicatrizes aparentes, cicatrizes alargadas, "umbigo em bola", escurecimento do umbigo, "umbigo estranho"; todas também percebidas pelo profissional, corroborando com as queixas apresentadas. Não houve nenhum

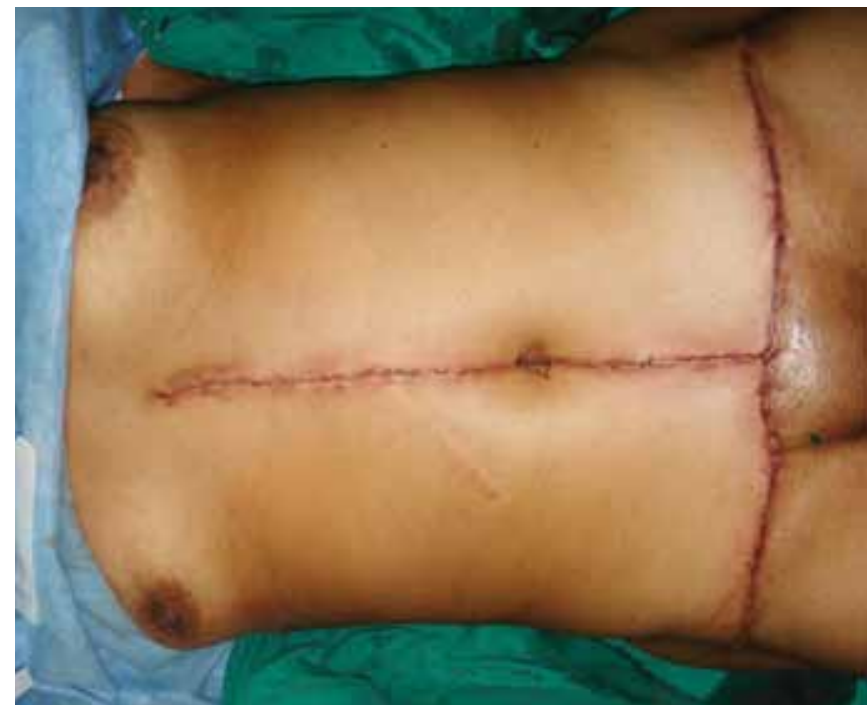

Figura 6 - Neoumbigo fixo à aponeurose, aspecto final da cirurgia.

relato de insatisfação com o umbigo por parte dos pacientes submetidos a neoumbilicoplastia. A única complicação apresentada em caso de neoumbigo foi uma deiscência total dos pontos de fixação, secundária a importante celulite de toda a ferida operatória, com formação de granulomas.

O posicionamento dos umbigos foi considerado adequado pelos pacientes e o examinador, independente da técnica utilizada.

Em relação à forma, foram observados umbigos mais verticalizados e de tamanho de pequeno a médio, em casos de neoumbigo (Figura 11), com uma discreta "cortina" superior, enquanto que umbigos circulares e largos, nas transposições umbilicais (Figura 12).

\section{DISCUSSÃO}

A cirurgia bariátrica vem estando cada vez mais próxima do cirurgião plástico e análises psicológicas quantitativas e qualitativas vêm mostrando uma melhora importante na qualidade de vida de seus pacientes ${ }^{13}$.

No estudo apresentado, as características gerais, dados transoperatórios e a incidência de complicações pós-operatórias são semelhantes aos da literatura ${ }^{14}$.

A dermolipectomia em âncora é técnica de eleição em grandes centros pós-cirurgia bariátrica e apresenta bons resultados, com curto tempo cirúrgico e baixo índice de complicações, principalmente quando o paciente consegue atingir o IMC mais próximo do ideal ${ }^{12}$. Colwell \& Borud $^{15}$ compararam as táticas de diversos autores e suas complicações, observando tendência a maiores complicações em cirurgias mais extensas de contorno corporal total. 

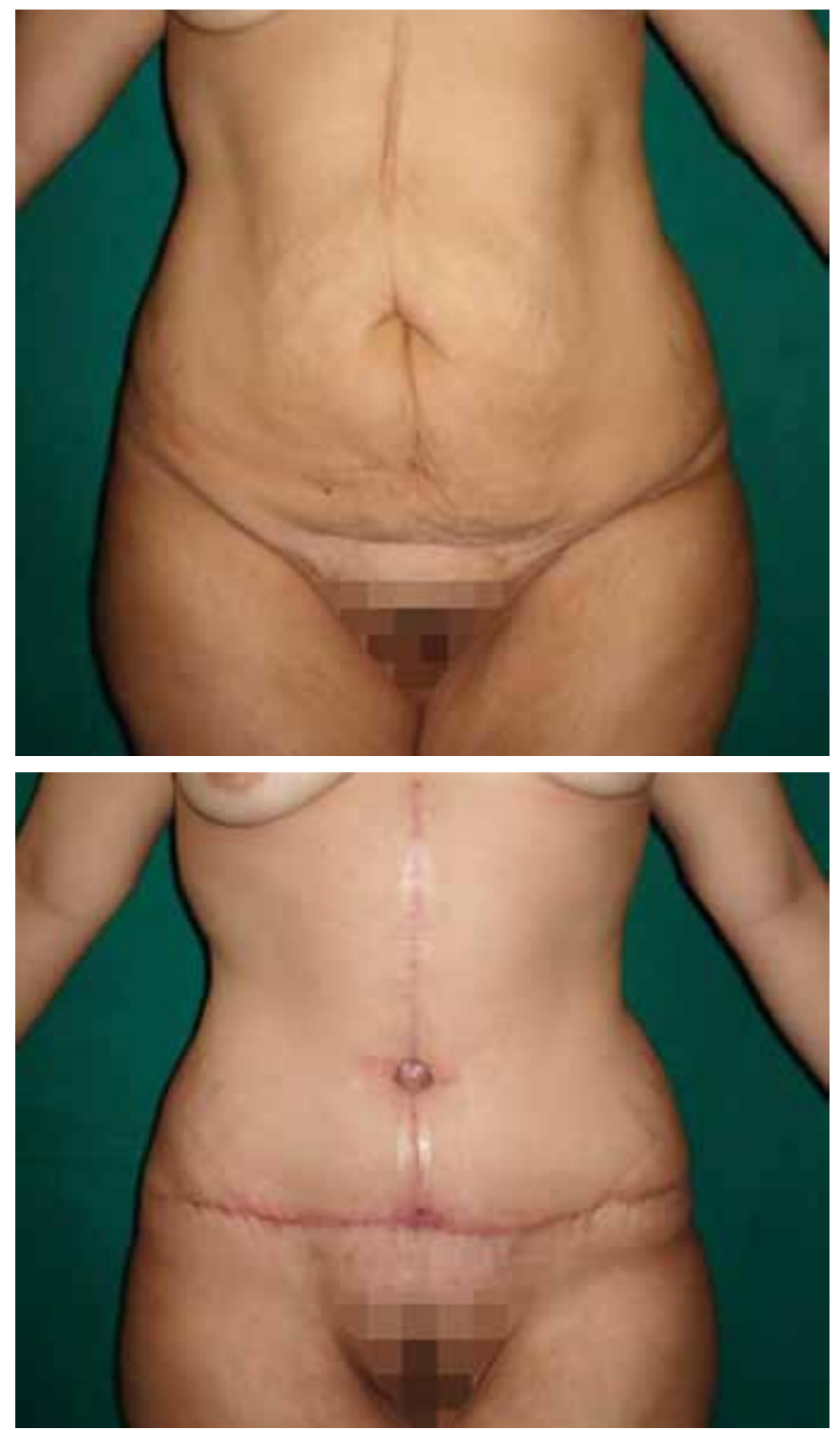

Figura 7 - Pré e pós-operatório de 3 meses de abdominoplastia em ancora + umbilicoplastia em losango.

Tabela 1 - Características gerais dos pacientes ex-obesos.

\begin{tabular}{l|c|c|c|c}
\hline & \multicolumn{2}{|c|}{$\begin{array}{c}\text { Neoumbilicoplastia } \\
(\mathbf{N}=17)\end{array}$} & \multicolumn{2}{c}{$\begin{array}{c}\text { Transposição } \\
\text { umbilical }(\mathbf{N}=14)\end{array}$} \\
\hline $\begin{array}{l}\text { Idade } \\
\text { (anos) }\end{array}$ & 33 a 55 & Média - 44 & 31 a 55 & Média - 46 \\
\hline Sexo & M (17\%) & F (83\%) & M (14\%) & F (86\%) \\
\hline $\begin{array}{l}\text { IMC } \\
\left(\mathrm{kg} / \mathrm{m}^{2}\right)\end{array}$ & 24 a 31,2 & $\begin{array}{c}\text { Média - } \\
28,3\end{array}$ & 21,6 a 31,1 & Média - 27 \\
\hline
\end{tabular}
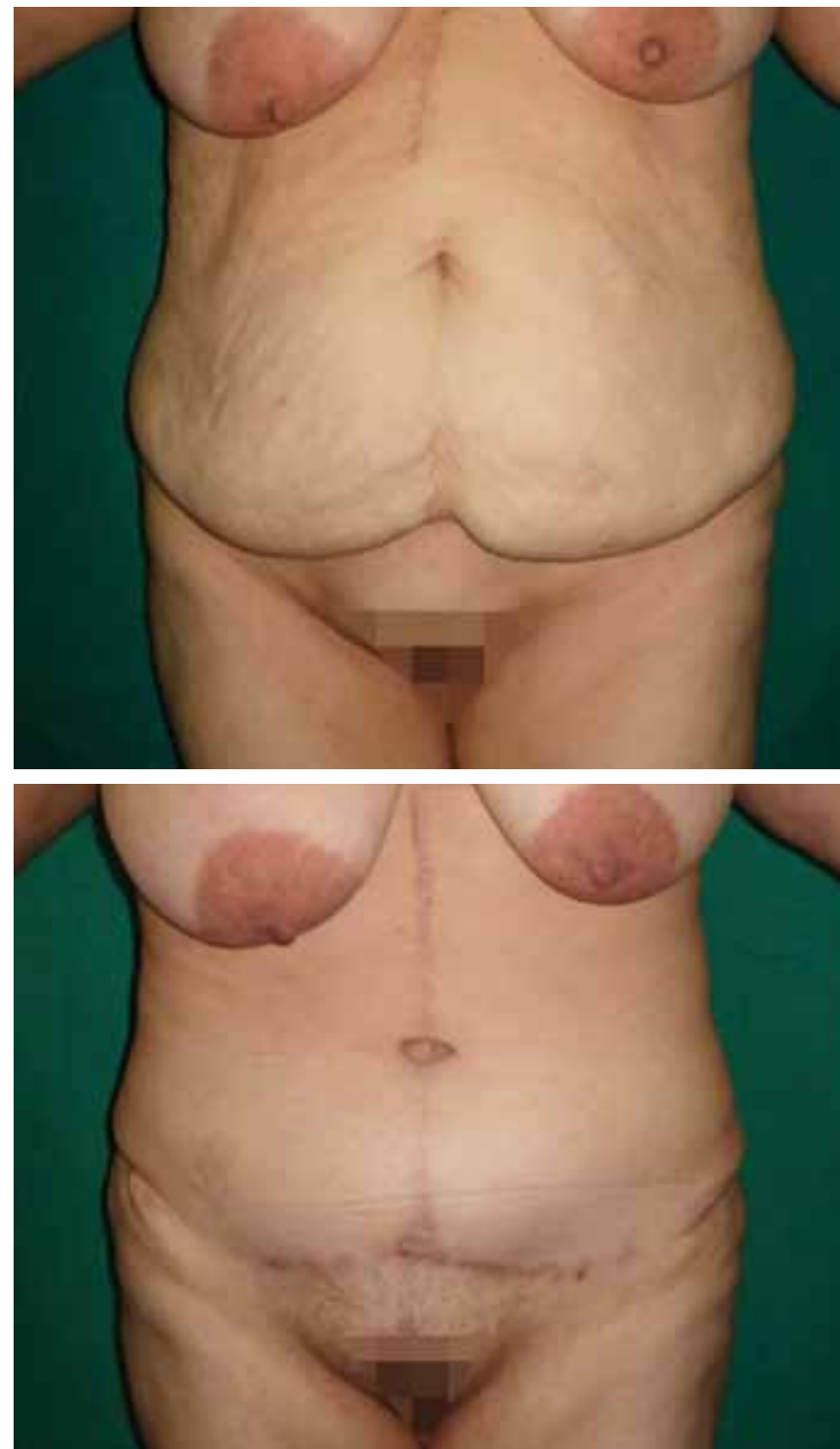

Figura 8 - Pré e pós-operatório de 1 ano e 3 meses de abdominoplastia em âncora + umbilicoplastia em losango.

Tabela 2 - Aspectos transoperatórios.

\begin{tabular}{l|c|c|c|c}
\hline & \multicolumn{2}{|c|}{$\begin{array}{c}\text { Neoumbilicoplastia } \\
(\mathbf{N}=17)\end{array}$} & \multicolumn{2}{c}{$\begin{array}{c}\text { Transposição } \\
\text { umbilical (N=14) }\end{array}$} \\
\hline $\begin{array}{l}\text { Peso da } \\
\text { peça (g) }\end{array}$ & $\begin{array}{c}1440 \mathrm{a} \\
8500\end{array}$ & $\begin{array}{c}\text { Médio - } \\
2600\end{array}$ & $\begin{array}{c}740 \mathrm{a} \\
3700\end{array}$ & $\begin{array}{c}\text { Médio - } \\
1990\end{array}$ \\
\hline $\begin{array}{l}\text { Tempo } \\
\text { transoperatório } \\
\text { (h) }\end{array}$ & $\begin{array}{c}1: 45 \mathrm{a} \\
3: 45\end{array}$ & $\begin{array}{c}\text { Médio - } \\
2: 25\end{array}$ & $\begin{array}{c}1: 35 \mathrm{a} \\
3: 15\end{array}$ & $\begin{array}{c}\text { Médio - } \\
2: 15\end{array}$ \\
\hline $\begin{array}{l}\text { Incidência } \\
\text { de hérnias }\end{array}$ & $56,2 \%$ & & $53,1 \%$ & \\
\hline Uso de tela & & & $1(7,1 \%)$ & \\
\hline
\end{tabular}



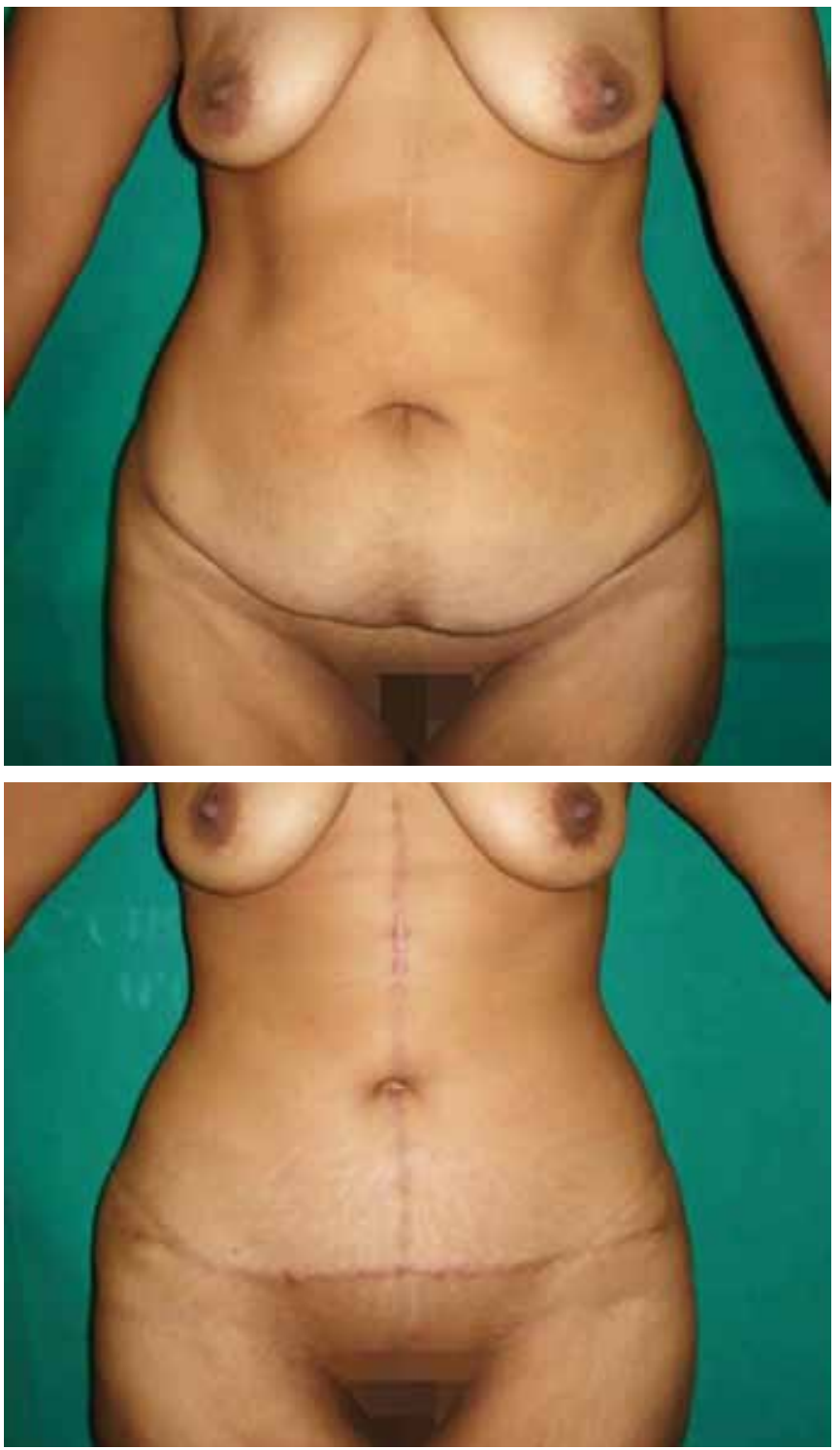

Figura 9 - Pré e pós-operatório de 6 meses de abdominoplastia em ancora + neoumbilicoplastia.

Já o umbigo, por muitas vezes, é um local que gera insatisfação nas dermolipectomias abdominais, independente da técnica da mesma.

Qualquer tipo de reconstrução umbilical que objetiva o bom resultado estético deve buscar algumas características importantes, como tamanho, forma, localização e profundidade adequadas. Ao mesmo tempo, na presença de uma cicatriz, o ideal seria que não ocorressem complicações, como estenose, cicatriz aparente, hipertrofias ou alargamentos. Há uma tendência para maior aceitação quando as cicatrizes peri-umbilicais são mais estreitas e menos visíveis ${ }^{16}$.
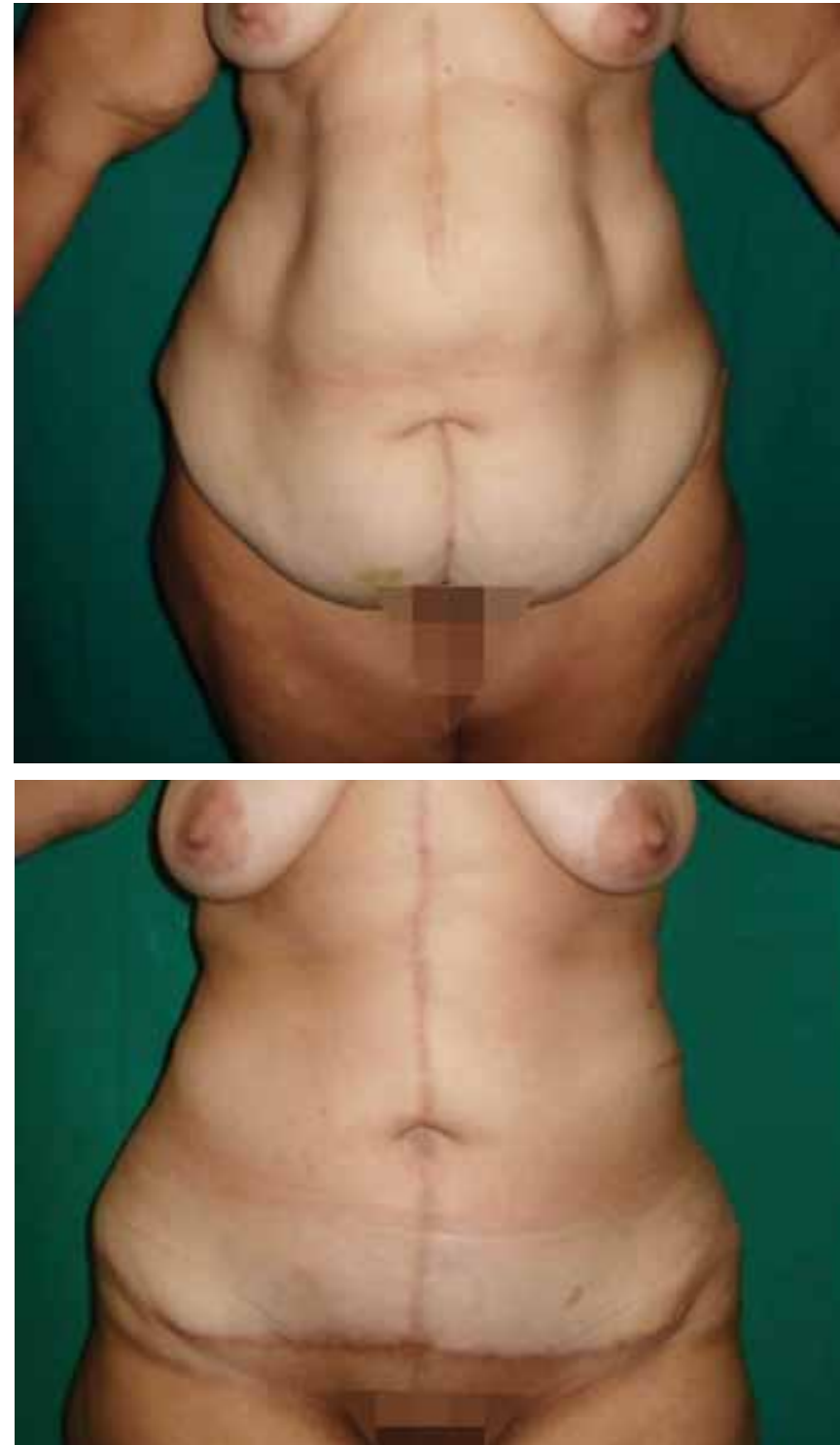

Figura 10 - Pré e pós-operatório de 9 meses de abdominoplastia em âncora + neoumbilicoplastia.

Quanto ao tamanho e forma, Craig et al. ${ }^{17}$ avaliaram umbigos de 147 mulheres e observaram que as melhores notas do ponto de vista estético foram àqueles que apresentavam um tamanho de médio a pequeno, um formato mais verticalizado, com a presença ou não de uma "cortina" de pele superiormente. Essas características foram obtidas em sua maioria nos casos de neoumbilicoplastia realizados.

Um estudo indiano avaliou 75 mulheres jovens com IMC médio de $29,9 \mathrm{~kg} / \mathrm{m}^{2}$ e concluiu que o umbigo estava posicionado numa altura média de proporção 1,6:1 entre o apêndice xifóide e a sínfise púbica. A profundidade média encontrada foi de $1,16 \mathrm{~cm}^{18}$. A associação dos pontos de 

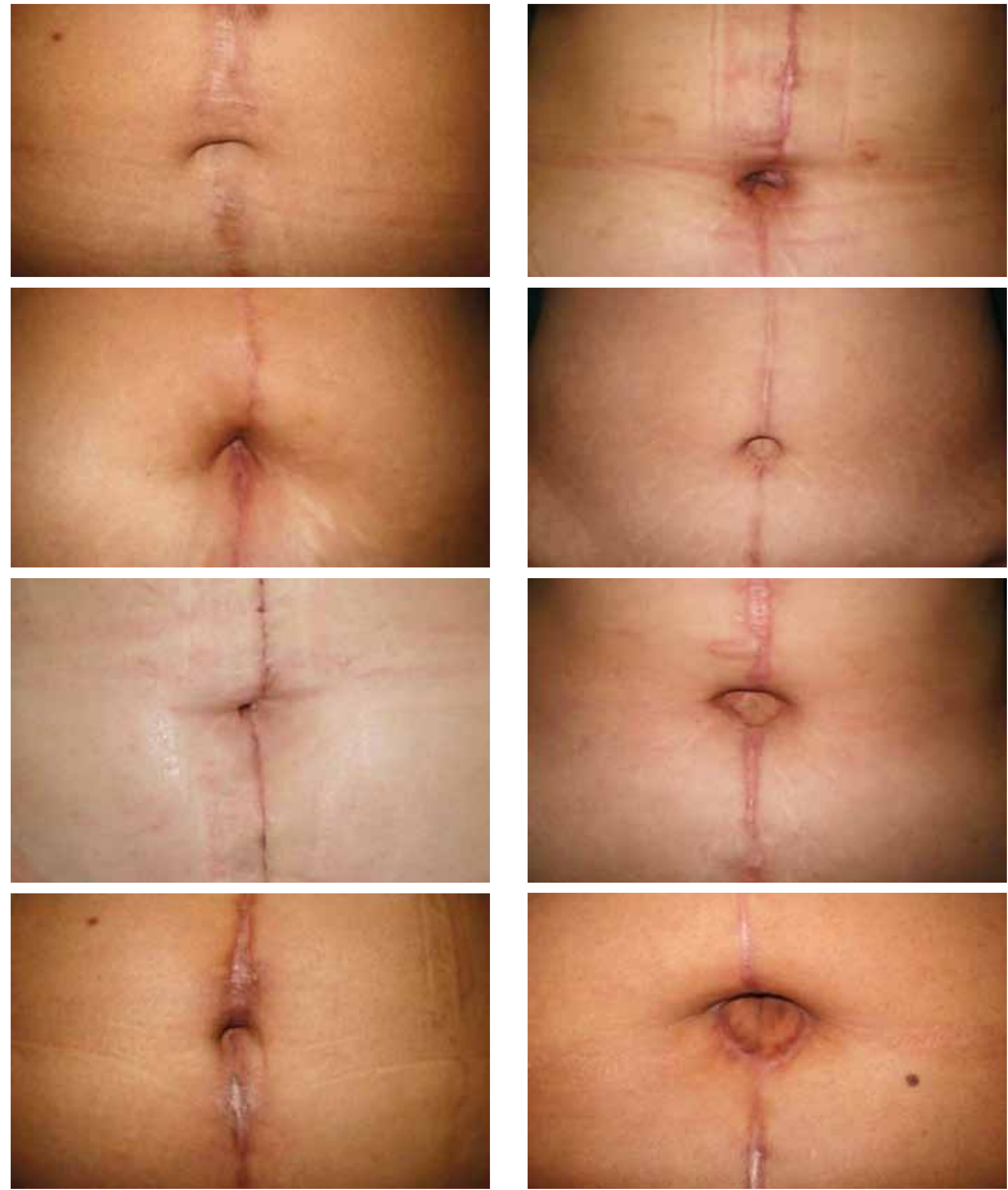

Figura 11 - Casos de neoumbilicoplastias realizadas no estudo.

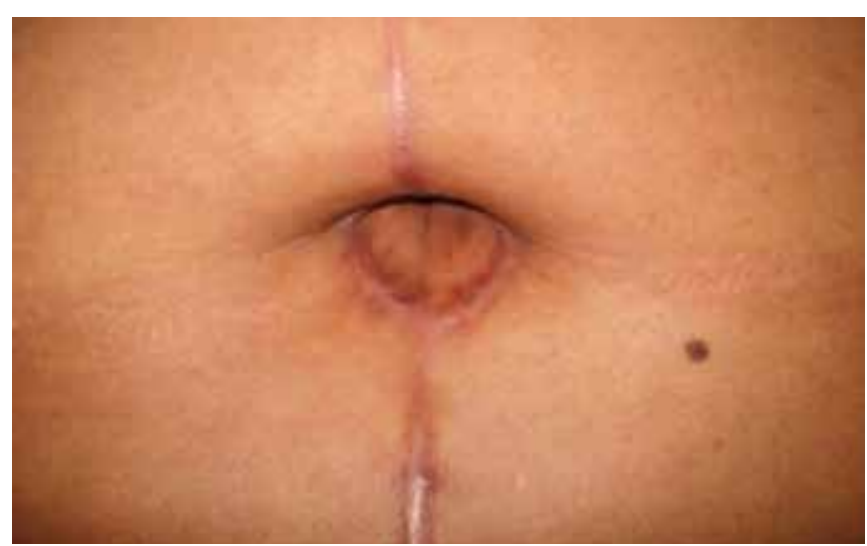

Figura 12 - Casos de umbilicoplastias de transposição realizadas no estudo. 
Tabela 3 - Complicações pós-operatórias.

\begin{tabular}{l|c|c|c|c}
\hline & \multicolumn{2}{|c|}{$\begin{array}{c}\text { Neoumbilicoplastia (N=17) } \\
\text { Incidência }\end{array}$} & $\begin{array}{c}\text { Transposição umbilical (N=14) } \\
\text { Incidência }\end{array}$ \\
\hline Seroma casos & $43 \%$ & 4 & $28 \%$ \\
\hline Deiscências de FO & 4 & $17,6 \%$ & 1 & $28 \%$ \\
\hline Celulite de FO & 3 & $11,7 \%$ & 5 & $7,1 \%$ \\
\hline Cicatrizes alargadas & 2 & $41 \%$ & 1 & $35,7 \%$ \\
\hline Hematoma & 7 & - & 2 & $7,1 \%$ \\
\hline Deiscência parcial/total do umbigo & 1 & $5,8 \%$ & 6 & $14,2 \%$ \\
\hline Despigmentação umbilical & 1 & $5,8 \%$ & & $42 \%$ \\
\hline Necrose cutânea dos retalhos & - & - & - & \\
\hline
\end{tabular}

referência das cristas ilíacas com a área do coto umbilical amputado mostrou-se suficiente e adequada na determinação do bom posicionamento umbilical encontrado nas neoumbilicoplastias realizadas. Quanto à profundidade, acreditamos que a boa fixação com fios inabsorvíveis dos retalhos do neoumbigo pôde garantir a manutenção da profundidade do mesmo no período estudado.

Diversas técnicas são descritas tentando se obter cicatrizes mais discretas associados a umbigos mais naturais ${ }^{16}$. Malic et al. ${ }^{19}$ compararam 2 técnicas de umbilicoplastia de transposição e obtiveram 15 a $58 \%$ de revisões cicatriciais, apesar de bons resultados. Quando se trata de pacientes ex-obesos, o pedículo desses cotos umbilicais é longo, de difícil manipulação e vascularização limítrofe, ocasionando, na opinião de pacientes e cirurgiões, piores resultados. A incidência de complicações especificamente do umbigo não é bem descrita na literatura, estando, assim subnotificada. Cortes et al. ${ }^{12}$ observaram $8 \%$ de sofrimento em 39 pacientes operados. A incidência encontrada de despigmentação de $42 \%$ e de deiscências de $14 \%$ nas umbilicoplastias convencionais corrobora com esses dados. Foi observada pelos pacientes e examinador tendência a cotos umbilicais arredondados e com cicatrizes mais visíveis.

\section{CONCLUSÃO}

A abdominoplastia em âncora, já bem citada na literatura, continua se apresentando como uma excelente opção de dermolipectomia em pacientes com grandes perdas ponderais e que têm uma cicatriz mediana associada, como a da gastroplastia.

A técnica de neoumbilicoplastia descrita nesse estudo se mostrou um método de reconstrução umbilical de fácil realização e de melhores resultados estéticos que a tática de umbilicoplastia por transposição, com menor incidência de complicações, importante satisfação do autor e dos pacientes. Há, porém, a necessidade de avaliação por meio de um estudo analítico e de longo prazo para que os resultados encontrados tenham significância estatística e possam ser extrapolados para o universo de pacientes ex-obesos.

O perfil e as características gerais avaliadas nos pacientes estudados são compatíveis com outros trabalhos publicados na literatura.

\section{REFERÊNCIAS}

1. Costa LF, Landecker A, Manta AM. Optimizing body contour in massive weight loss patients: the modified vertical abdominoplasty. Plast Reconstr Surg. 2004;114(7):1917-23.

2. Persichetti P, Simone P, Scuderi N. Anchor-line abdominoplasty: a comprehensive approach to abdominal wall reconstruction and body contouring. Plast Reconstr Surg. 2005;116(1):289-94.

3. Delerm A. Refinements in abdominoplasty with emphasis on reimplantation of the umbilicus. Plast Reconstr Surg. 1982;70(5):632-7.

4. Craig SB, Faller MS, Puckett CL. In search of the ideal female umbilicus. Plast Reconstr Surg. 2000;105(1):389-92.

5. Akbas H, Guneren E, Eroglu L, Uysal OA. Natural-looking umbilicus as an important part of abdominoplasty. Aesthetic Plast Surg. 2003;27(2):139-42.

6. Lee MJ, Mustoe TA. Simplified technique for creating a youthful umbilicus in abdominoplasty. Plast Reconstr Surg. 2002;109(6):2136-40.

7. Baack BR, Anson G, Nachbar JM, White DJ. Umbilicoplasty: the construction of a new umbilicus and correction of umbilical stenosis without external scars. Plast Reconstr Surg. 1996;97(1):227-32.

8. Yotsuyanagi T, Nihei Y, Sawada Y. A simple technique for reconstruction of the umbilicus, using two twisted flaps. Plast Reconstr Surg. 1998;102(7):2444-6.

9. Sevin A, Sevin K, Senen D, Erdogan B. A new method for umbilicus reconstruction: preliminary report. Aesthetic Plast Surg. 2006;30(5):589-91.

10. Masuda R, Takeda A, Sugimoto T, Ishiguro M, Uchinuma E. Reconstruction of the umbilicus using a reverse fan-shaped flap. Aesthetic Plast Surg. 2003;27(5):349-53. 
11. Franco D, Medeiros J, Farias C, Franco T. Umbilical reconstruction for patients with a midline scar. Aesthetic Plast Surg. 2006;30(5):595-8.

12. Cortes JES, Oliveira DP, Sperly A. Abdominoplastias em âncora em pacientes ex-obesos. Rev Bras Cir Plást. 2009;24(1):57-63.

13. Cintra Junior W, Modolin M, Gobbi CIC, Gemperli R, Ferreira MC. Abdominoplastia circunferencial em pacientes após cirurgia bariátrica: avaliação da qualidade de vida pelo critério adaptativo. Rev Bras Cir Plást. 2009;24(1):52-6.

14. Capella JF. Lifting corporal. In: Aly A, ed. Cirurgia do contorno corporal após grandes perdas ponderais: cirurgia plástica. Rio de Janeiro: Dilivros; 2008. p.33-61.
15. Colwell AS, Borud LJ. Optimization of patient safety in postbariatric body contouring: a current review. Aesthet Surg J. 2008;28(4):437-42.

16. Rosique MJF, Rosique RG, Lee FDI, Kawakami H, Glattstein N, Mélega JM. Estudo comparativo entre técnicas de onfaloplastia. Rev Bras Cir Plást. 2009;24(1):47-51.

17. Craig SB, Faller MS, Puckett CL. In search of the ideal female umbilicus. Plast Reconstr Surg. 2000;105(1):389-92.

18. Abhyankar SV, Rajguru AG, Patil PA. Anatomical localization of the umbilicus: an Indian study. Plast Reconstr Surg. 2006;117(4):1153-7.

19. Malic CC, Spyrou GE, Hough M, Fourie L. Patient satisfaction with two different methods of umbilicoplasty. Plast Reconstr Surg. 2007;119(1):357-61.

Correspondência para:

Ernando Luiz Ferraz Cavalcanti

Rua Caio Pereira, 30, apto. 1301 - Rosarinho - Recife, PE, Brasil - CEP 52041-010

E-mail: ernandolfc@hotmail.com 